\title{
Quiste epidermoide testicular: una rara entidad de difícil diagnostico preoperatorio
}

\author{
C. Aguilera Tubet, G. López Rasines*, A. Roca Edreira, B. Martín García, \\ R. Hernández Rodríguez, J.A Portillo Martín, J.L. Gutiérrez Baños, M.A. Correas Gómez, \\ J.I. Del Valle Schaan, M.A Rado Velázquez, F. Ruiz Izquierdo, R. Ballestero Diego
}

Servicio de Urología y *Servicio de Radiodiagnóstico. Hospital Universitario Marqués de Valdecilla. Santander.

Actas Urol Esp 2005; 29 (9): 905-908

\section{RESUMEN \\ QUISTE EPIDERMOIDE TESTICULAR: UNA RARA ENTIDAD DE DIFÍCIL DIAGNOSTICO PREOPERATORIO}

Los quistes epidermoides intratesticulares son tumores raros, constituyendo el $1 \%$ de todos los tumores testiculares. Se trata de tumoraciones benignas que plantean un difícil diagnóstico diferencial preoperatorio frente a los tumores malignos testiculares. La ausencia de elevación de los marcadores tumorales y la apariencia ecográfica, pueden orientar hacia su diagnóstico preoperatorio y en este caso la cirugía conservadora del testículo.

Se presenta el caso de un paciente de 22 años que consulta por una masa en el testículo izquierdo. En este caso, el diagnóstico ecográfico preoperatorio no descartaba otras patologías por lo que se procedió a la realización de orquiectomía inguinal izquierda.

Palabras clave: Quiste epidermoide, ecografía, testículo

\begin{abstract}
TESTICULAR EPIDERMOID CYST: UNCOMMON LESION OF DIFFICULT PREOPERATIVE DIAGNOSIS

Intratesticular epidermoid cysts are rare tumours that constitute one percent of all testicular masses. They are bening lesions that make differential diagnosis from malignant testicular tumours difficult. The absence of serum markers elevation and ultrasound imaging could support these lesions being bening epidermoid cysts, and in that case, conservative surgery is adequate.

We present the case of a 22 years old patient who complains of a left testicular mass. In this case ultrasound diagnosis was non-specific and a left radical inguinal orchiectomy was performed.
\end{abstract}

Keywords: Epidermoid cyst, ultrasound, testicle.

$\mathrm{E}^{1}$ quiste epidermoide de testículo es una entidad benigna poco frecuente que suele aparecer en varones jóvenes. Su curso clínico le hace indistinguible de otras lesiones malignas testiculares, aunque se crecimiento suele ser mas lento. Presenta una imagen ecográfica característica aunque no patognomónica en capas de cebolla o en diana que se corresponde con su patrón anatomopatológico. El tratamiento de elección es la tumorectomía con conservación del teste restante aunque en caso de duda se ha de proceder a la orquiectomía radical por vía inguinal.

\section{CASO CLÍNICO}

Se presenta el caso de un varón de 22 años que acude al Servicio de Urgencias tras notar una masa dura en testículo izquierdo mediante autoexploración. Como único antecedente previo destacable, amigdalectomía en la infancia. No refiere historia previa de traumatismo ni proceso inflamatorio en la zona. A la exploración se pone en evidencia una masa dura e indolora a nivel del polo superior del testículo izquierdo. La transiluminación resultó negativa y el resto de la exploración fue normal, no encontrándose masas a 
nivel abdominal ni ginecomastia. Es dado de alta con tratamiento antiinflamatorio y antibiótico en espera de la realización de ecografía testicular de forma ambulatoria. Con posterioridad, el paciente acude a otro centro donde se realiza ecografía testicular que es informada como imagen nodular sugestiva de seminoma.

La ecografia realizada en nuestro centro revela una imagen nodular en el testículo izquierdo, de $19 \mathrm{~mm}$ de diámetro mayor, sólida, con bandas ecogénicas en capas en su interior y pequeños focos ecogénicos que probablemente correspondan a calcio, hallazgos que se pueden asociar a los quistes epidermoides si bien no se pueden descartar otras causas (Fig.1).

Tanto los marcadores tumorales (alfa-fetoproteína y $\beta-\mathrm{HCG}$ ) como la radiografía de tórax fueron normales.

Ante la duda razonable preoperatoria de tratarse de una tumoración maligna se procedió a la realización de orquiectomía izquierda por vía inguinal, siguiendo los criterios de la cirugía oncológica.

$\mathrm{El}$ análisis histológico postoperatorio revela un testículo izquierdo de $5 \times 4 \times 2 \mathrm{~cm}$. La superficie albuginea presenta una zona blanquecina que contrasta con el resto, brillante y azulada. Dicha zona parece corresponder en cortes seriados con una lesión nodular localizada en el polo superior de $2.2 \times 1.8 \times 2.2 \mathrm{~cm}$. Es de coloración blanquecina y presenta un aspecto en capas de cebolla. El resto del parénquima testicular es normal no existiendo elementos teratomatosos ni neoplasia intratubular en el tejido adyacente.

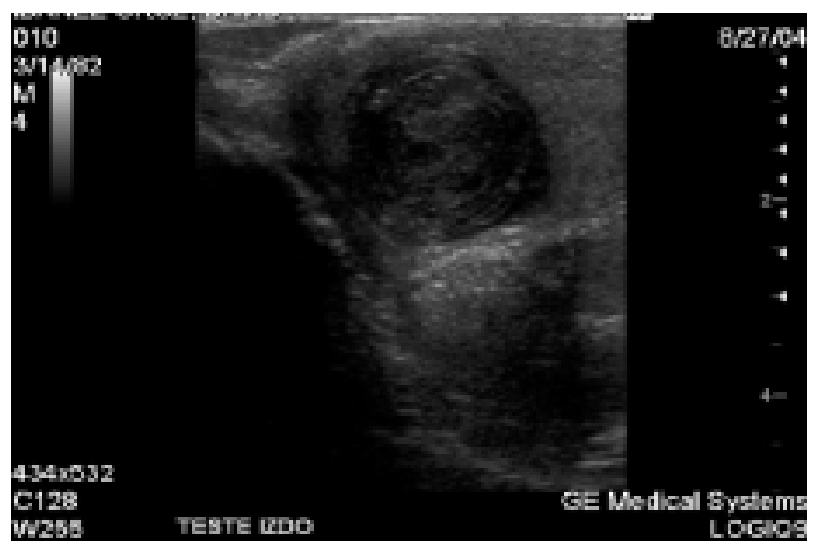

FIGURA 1. Ecografia de testiculo izquierdo mostrando una lesión con un anillo hipoecoico y la tipica imagen interna en capas de cebolla.

\section{DISCUSIÓN}

El quiste epidermoide del testículo es una rara entidad benigna que corresponde al $1 \%$ de todos los tumores intratesticulares ${ }^{1}$. Los quistes epidermoides son frecuentes en otros órganos de la economía pero constituyen una excepción en su localización testicular. Se trata de lesiones solitarias cuya edad de aparición se sitúa entre la segunda y la cuarta década de la vida; aunque su aparición en niños es más infrecuente, también se han descrito casos. La lesión típicamente se presenta como un nódulo testicular pequeño e indoloro detectado mediante autoexploración o por una exploración médica motivada por otra causa, subyacente a la albugínea. No obstante, la exploración física no diferencia estas lesiones de otros tumores malignos de aparición más frecuente. Es más frecuente encontrarla en el testículo derecho, en hombres blancos y en testículos con antecedentes de criptorquidia. Su presentación bilateral es rara ${ }^{2}$.

Los estudios con marcadores tumorales (AFP y Beta-HCG) resultan normales, aunque existe un caso en la literatura de un falso incremento de Beta-HCG en un caso diagnosticado de quiste epidermoide, debido a anticuerpos anormales ${ }^{3}$.

La histogénesis del quiste epidermoide no es del todo conocida. Algunos autores ${ }^{4,5}$ han considerado el quiste epidermoide como el resultado de un teratoma que ha sufrido sólo diferenciación monodermal porque contiene sólo la parte epidérmica del ectodermo. La ausencia de neoplasia intraepitelial testicular, el precursor histológico del tumor de células germinales, en el testículo afecto plantea dudas acerca del origen germinal del quiste epidermoide. Otras razones que han llevado a cuestionar su condición teratomatosa, son que el comportamiento biológico del quiste epidermoide difiere mucho del de el teratoma; tasa de crecimiento más lenta y la ausencia de recidiva local y desarrollo de metástasis ${ }^{4}$. Por todo esto se considera al quiste epidermoide como una lesión benigna, aunque en la literatura se ha descrito el caso de un seminoma desarrollado después del tratamiento conservador de un quiste epidermoide ${ }^{6}$.

El estudio ecográfico permite diferenciar entre lesiones intratesticulares y extratesticulares, definiendo las lesiones sólidas y quísticas y per- 
mitiendo el despistaje de lesiones múltiples. No obstante la ecografía testicular no permite diferenciar lesiones benignas de malignas. Los quistes epidermoides suelen mostrarse como lesiones bien delimitadas en el parénquima testicular, de carácter heterogéneo, generalmente con centro hiperecoico (queratina) y anillo periférico hipoecoico (capa escamosa o fibrosa). Los quistes epidermoides se diferencian bien de los quistes simples debido a la presencia de detritus en su interior. Con la ecografía doppler testicular se demuestra la ausencia de flujo sanguíneo en todo el quiste epidermoide ${ }^{5,7,8}$. Para algunos autores la ecografia testicular no es especifica de quiste epidermoide, mientras que para otros el patrón ecográfico con aspecto laminado en capas de cebolla o en diana son muy sugestivos de esta patología, permitiendo una cirugía conservadora. La RMN muestra también una imagen típica en ojo de buey o en diana ${ }^{9,10}$. Aun así, la variabilidad (Tabla I) existente en los hallazgos ecográficos sugiere precaución a la hora de etiquetar la lesión como benigna en la ecografía ${ }^{7}$.

El diagnóstico de quiste epidermoide lo da el análisis histológico. Price estableció cinco criterios para confirmar el diagnóstico de una lesión como quiste epidermoide benigno testicular ${ }^{11}$ :

1) quiste localizado del parénquima testicular;

2) el centro del quiste está sustituido por material amorfo o restos queratinizados;

3) la pared quística es un tejido fibroso con cubierta completa o incompleta de tejido escamoso;

4) no hay elementos anexales dérmicos o teratomatosos dentro del quiste o en algún lugar dentro del testículo;

5) no se observa cicatriz dentro del parénquima restante. Pueden observarse eventuales reacciones inflamatorias lipogranulomatosas con calcificaciones focales y la pared quística debe encontrarse separada de la albugínea. Es importante la ausencia de cicatrices en el parénquima circundante ya que estas pueden representar células germinales malignas fundidas.

El diagnóstico preoperatorio es difícil. En varones con teste único, tumor bilateral o ante la sospecha ecográfica de quiste epidermoide con marcadores tumorales negativos, se debe proce-
Tabla I

Variabilidad de los hallazgos ecográficos.

\section{Aspecto ecográfico:}

1. diana

2. masa bien delimitada con anillo de calcificación

3. masa sólida con anillo ecogénico

4. aspecto en capas de cebolla

der a la enucleación de la lesión con preservación del teste restante. La base para la realización de esta cirugía conservadora se encuentra en que son pacientes jóvenes, en los que se debe preservar al máximo su fertilidad, en los que puede haber también implicaciones psicológicas y en los que no se ha demostrado hasta la fecha evidencia de metástasis.

La exploración quirúrgica debe seguir los principios de cirugía oncológica, siendo ésta por vía inguinal, con clampaje previo del cordón, con protección mediante paños de la herida, y se aconseja la enucleación total de la lesión y no su biopsia. El estudio peroperatorio por congelación de la pieza de tumorectomía y dos muestras de tejido testicular adyacente ha demostrado su fiabilidad en el diagnostico de estas lesiones. Si la biopsia detecta elementos teratomatosos en la pared del quiste o carcinoma in situ del epitelio seminífero se realiza reexploración y orquiectomía radical $^{12,13}$. En el caso de dudas en el diagnóstico preoperatorio de quiste epidermoide, se debe proceder a la orquiectomía por vía inguinal como ocurrió en el caso que exponemos.

\section{REFERENCIAS}

1. Moghe PK, Brady AP.Ultrasound of testicular epidermoid cysts. Br J Radiol 1999;72(862):942-945.

2. Sloan JC, Beck SD, Bihrle R, Foster RS.: Bilateral testicular epidermoid cysts managed by partial orchiectomy. J Urol 2002; 167(1):255-256.

3. Mills Jn, Nguyen Tt, Williams Rd. Falsely increased betahuman chorionic gonadotropin with a testicular epidermoid cyst. J Urol 2001;166(6):2314.

4. Reinberg Y, Manivel JC, Llerena J, Niehans G, Fraley E. Epidermoid cyst (monodermal teratoma) of the testis. Br J Urol 1990;66:648-651.

5. Malvica RP.: Epidermoid cyst of the testicle: an unusual sonographic finding. Am J Roentgenol. 1993;160(5):10471048.

6. Woo LL, Curtis MR, Cohen MB, Sandlow JI. Development of seminoma following conservative treatment of testicular epidermoid cyst. J Urol 2001;165(5):1635-1636. 
7. Dogra VS, Gottlieb RH, Rubens DJ, Oka M, Di Sant Agnese AP. Testicular epidermoid cysts: Sonographic features with histopathologic correlation. Journal of clinical ultrasound 2001;29(3):192-196.

8. Bahnson RR, Slasky BS, Ernstoff MS, Banner BF. Sonographic characteristics of epidermoid cyst of testicle. Urology 1990;35(6):508-510.

9. Cho JH, Chang JC, Park BH, Lee JG, Son CH. Sonographic and MR imaging findings of testicular epidermoid cysts. Am J Roentgenol 2002;178(3)743-748.

10. Langer JE, Ramchandani P, Siegelman ES, Banner MP.: Epidermoid cysts of the testicle : sonographic and MR imaging features. Am J Roentgenol 1999;173(5):1295-1296.

11. Price EB.: Epidermoid cysts of the testis. A clinical and pathological analysis of 69 cases from the Testicular Tumor Registry. J Urol 1969;102:708-713.
12. Walsh C, Rushton HG.: Diagnosis and management of teratomas and epidermoid cysts. Urol Clin North Am 2000; 27(3):509-518.

13. MartÍnez VM, Cruceyra Betriou G, Piedra Lara J.D, Capitan Majon C, Carrero Lopez VM, Leiva Galvis O.: Quiste epidermoide testicular a propósito de un nuevo caso y revisión de la literatura. Arch. Esp. Urol 2004;57: 641-643.

Dra. C. Aguilera Tubet

Paseo de Canalejas 11D 7ํㅜㄱ, 39004

Santander, Cantabria

(Trabajo recibido el 18 de febrero 2005) 